\title{
Elastic Constants and Properties of B2-type FeAl and Fe-Cr-Al Alloys from First-Principles Calculations
}

\author{
Y. CHEN ${ }^{1,2, a, *}$, Z. J. YAO ${ }^{1}$, P. Z. ZHANG ${ }^{1}$, X. X. LUO ${ }^{1}$, Z. L. ZHANG ${ }^{1}$ and P. D. \\ $\mathrm{HAN}^{3}$ \\ ${ }^{1}$ College of materials science \& Engineering, Nanjing University of Aeronautics and Astronautics, \\ Nanjing 211106, China \\ ${ }^{2}$ School of computer \& software, Nanjing Institute of Industry Technology, Nanjing 210046, China \\ ${ }^{3}$ College of Materials Science and Engineering, Taiyuan University of Technology, Taiyuan 030024 , \\ China \\ achiniku@qq.com \\ ${ }^{*}$ Corresponding author
}

Keywords: FeAl Intermetallic Compounds, Density Functional Theory, Strain-Stress Method, Elastic Constants.

\begin{abstract}
The elastic constants $\left(c_{i j}\right)$ and elastic properties of B2-phase FeAl intermetallic compounds and $\mathrm{Fe}-\mathrm{Cr}-\mathrm{Al}$ ternary alloys are studied by performing first-principles density functional theory calculations. The stable structures of $\mathrm{Fe}-\mathrm{Cr}-\mathrm{Al}$ and the elastic constants of $\mathrm{FeAl}$ and $\mathrm{Fe}-\mathrm{Cr}-\mathrm{Al}$ are predicted within the local density approximation (LDA) and PBE formulations of the generalized gradient approximation (GGA). It is found that more precise calculations of the $c_{i j}$ values of $\mathrm{Fe}-\mathrm{Cr}-\mathrm{Al}$ can be performed using the GGA-PBE method by comparing with experimental results. The predicted $c_{i j}$ values of $\mathrm{Fe}-\mathrm{Cr}-\mathrm{Al}$ will help in predicting the elastic properties of $\mathrm{Fe}-\mathrm{Cr}-\mathrm{Al}$. The electronic structures of $\mathrm{FeAl}$ and $\mathrm{Fe}-\mathrm{Cr}-\mathrm{Al}$ are analyzed to determine the micro mechanism of the two compounds.
\end{abstract}

\section{Introduction}

Important signatures of $\mathrm{Fe}-\mathrm{Al}$ intermetallic compounds are heat corrosion and erosion resistance, high-temperature oxidation resistance, sulfidation, and low cost. However, the shortage of room-temperature ductility and low high-temperature strength of FeAl hinder its industrial use. ${ }^{[1]}$ Alloying is a stable method used to intensify room-temperature ductility and strength of iron aluminides. $\mathrm{Cr}$ is one of the most popular alloy elements for solid-solution strengthening. ${ }^{[2,3]}$ It is essential to obtain the elastic stiffness constants (cij) of $\mathrm{FeAl}$ and $\mathrm{Fe}-\mathrm{Cr}-\mathrm{Al}$ to defermine the $\mathrm{Cr}$ alloying effects on the mechanical properties of iron aluminides.

In this work, the $c_{i j}$ values of $\mathrm{B} 2-\mathrm{FeAl}$ and $\mathrm{Fe}-\mathrm{Cr}-\mathrm{Al}$ were calculated based on first-principles. The cohesive energies were calculated for structures with the alloying $\mathrm{Cr}$ replacing both $\mathrm{Fe}$ and $\mathrm{Al}$ atoms to determine the preferred occupation sites of $\mathrm{Cr}$ in FeAl. The elastic modulus calculated by $c_{i j}$, such as the Pugh ratio, the Poisson's ratio, and the Cauchy pressure, can be used to forecast the elastic properties of the two compounds. Based on the values obtained, the accuracy of the calculation method can be determined.

\section{Methodology}

Calculations were performed using the Cambridge Sequential Total Energy Package (CASTEP) ${ }^{[4]}$ based on the density functional theory. Both the generalized gradient approximation (GGA) PBE scheme ${ }^{[5]}$ and the local density approximation (LDA) ${ }^{[6]}$ were employed as the exchange correlation functions. Lattice parameter optimizations were performed using the Broyden-Fletcher-Goldfarb-Shanno (BFGS) method. The ultra soft pseudo-potential was used as the all atomic pseudo-potential. The cut-off energy was set to $420 \mathrm{eV}$, and the k-points were set to 22 
$\times 22 \times 22$. The total energy convergence was set to $1.0 \times 10-5 \mathrm{eV} \cdot$ atom- 1 , the force acting convergence was set to $0.05 \mathrm{eV} \cdot \AA-1$, the stress deviation was set to $0.1 \mathrm{GPa}$, and the maximum displacement convergence was set to $0.002 \AA$. The spin polarization was performed.

\section{Results and Discussions}

\section{Effect of Cr on Structural Stability}

The FeAl has a symmetrical structure belonging to the Pm-3m space group. The 16-atom supercell model was adopted in this work containing $2 \times 2 \times 2 \mathrm{FeAl}$ unit cells, denoted as Fe8Al8. The crystal structure models of FeAl, Fe8CrAl7, and Fe7CrAl8 are shown in Fig.1. The cohesive energies were calculated to predict the substituting location of $\mathrm{Cr}$. The calculation equation for $\mathrm{Fe}-\mathrm{Cr}-\mathrm{Al}$ is by Eq. (1), in which every energy is the static energy calculated after the geometric optimization. In Eq. (1), Ecoh (FeaCrbAlc) refers to the cohesive energy of Fe-Cr-Al; Etot (FeaCrbAlc) refers to the total energies of $\mathrm{Fe}-\mathrm{Cr}-\mathrm{Al}, \mathrm{E}(\mathrm{Fe}), \mathrm{E}(\mathrm{Cr})$, and $\mathrm{E}(\mathrm{Al})$ respectively refer to the energies of $\mathrm{Fe}, \mathrm{Cr}$, and $\mathrm{Al}$, respectively. Moreover, a and b refer to the numbers of $\mathrm{Fe}$, and $\mathrm{Al}$ atoms, respectively, and the $\mathrm{Cr}$ atom number in the supercell is 1.

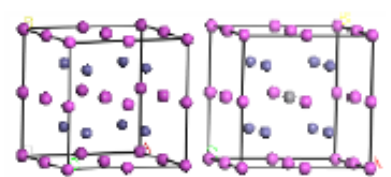

(a)

(b)

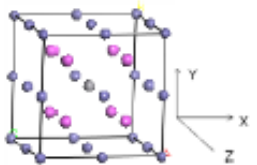

(c)

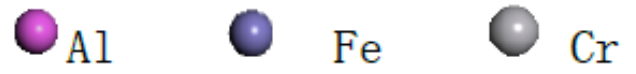

Figure1. Crystal structure models of the (a) FeAl supercell $\mathrm{Fe}_{8} \mathrm{Al}_{8}$, (b) $\mathrm{Fe}_{8} \mathrm{CrAl}_{7}$ and (c) $\mathrm{Fe}_{7} \mathrm{CrAl}_{8}$

$$
E_{c o h}\left(\mathrm{Fe}_{\mathrm{a}} \mathrm{CrAl} \mathrm{b}\right)=\frac{E_{\text {tot }}\left(\mathrm{Fe}_{\mathrm{a}} \mathrm{CrAl} \mathrm{b}\right)-\mathrm{a} E(\mathrm{Fe})-E(\mathrm{Cr})-\mathrm{b} E(\mathrm{Al})}{\mathrm{a}+1+b}
$$

Table 1 shows the cohesive energy of the replaced atom in the structures of supercells in which $\mathrm{Cr}$ is substituted for a Fe or $\mathrm{Al}$ atoms, calculated by GGA-PBE and LDA. These results illustrate that the structures in which $\mathrm{Cr}$ is substituted for an $\mathrm{Al}$ atom in the supercell are the more stable. Hence, the $\mathrm{Fe}_{8} \mathrm{CrAl}_{7}$ model was used in the following calculations. In the model the $\mathrm{Cr}$ centration is 6.25 at\%.

Table 1Cohesive energy (eV) and replaced atom.

\begin{tabular}{|c|c|c|c|}
\hline \multirow[b]{2}{*}{ Phase } & \multicolumn{2}{|c|}{ Cohesive Energy (eV) } & \multirow{2}{*}{$\begin{array}{l}\text { Replace } \\
\text { d Atom }\end{array}$} \\
\hline & GGA-PBE & LDA & \\
\hline $\mathrm{Fe}_{8} \mathrm{CrAl}$ & -7.34 & -5.101 & $\mathrm{Al}$ \\
\hline $\begin{array}{l}\mathrm{Fe}_{7} \mathrm{CrAl} \\
8\end{array}$ & -6.952 & -5.02 & $\mathrm{Fe}$ \\
\hline
\end{tabular}

\section{Effect of Cr on the Elastic Modulus}

The efficient strain-stress method ${ }^{[7]}$ was used to predict the elastic constants of FeAl and $\mathrm{Fe}-\mathrm{Cr}-\mathrm{Al}$ crystals. A strain $\boldsymbol{\varepsilon}$ is imposed on a crystal with lattice vector R, and the produced stressis $\boldsymbol{\sigma}$. The strain $\boldsymbol{\varepsilon}$ and stress $\boldsymbol{\sigma}$ are both tensors composed of nine components. However, because the strain and stress tensors have symmetry, they can be expressed in Voigt notation as Eqs. (2) and (3):

$$
\boldsymbol{\varepsilon}=\left(\begin{array}{ccc}
\varepsilon_{1} & \varepsilon_{6} / 2 & \varepsilon_{5} / 2 \\
\varepsilon_{6} / 2 & \varepsilon_{2} & \varepsilon_{4} / 2 \\
\varepsilon_{5} / 2 & \varepsilon_{4} / 2 & \varepsilon_{3}
\end{array}\right)
$$




$$
\boldsymbol{\sigma}=\left(\begin{array}{lll}
\sigma_{1} & \sigma_{6} & \sigma_{5} \\
\sigma_{6} & \sigma_{2} & \sigma_{4} \\
\sigma_{5} & \sigma_{4} & \sigma_{3}
\end{array}\right)
$$

The $3 \times 3$ deformed matrix $\mathbf{R}^{\prime}$ is obtained as $\mathbf{R}^{\prime}=\mathbf{R}(\mathbf{I}+\boldsymbol{\varepsilon})$, in which $\mathbf{R}$ is the undeformed vector, $\mathbf{I}$ is the $3 \times 3$ unit matrix , and $\boldsymbol{\varepsilon}$ is the strain matrix expressed as Eq. (2). $\mathbf{R}^{\prime}$ is expressed as Eq. (4):

$$
\mathbf{R}^{\prime}=\mathbf{R}\left(\begin{array}{lll}
1+\varepsilon_{1} & \varepsilon_{6} / 2 & \varepsilon_{5} / 2 \\
\varepsilon_{6} / 2 & 1+\varepsilon_{2} & \varepsilon_{4} / 2 \\
\varepsilon_{5} / 2 & \varepsilon_{4} / 2 & 1+\varepsilon_{3}
\end{array}\right)
$$

The generalized Hooke's law can be expressed as in Eq. (5).

$$
\sigma_{i=} C_{i j} \varepsilon_{j}
$$

In Eq. (5), $C_{i j}$ is an element of the $6 \times 6$ elastic constant matrix. Eq (6) is the expansion of Eq. (5):

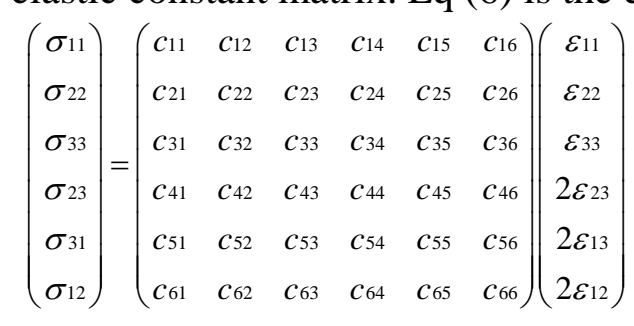

In the cubic crystal structure, symmetric relations such as $C_{11}=C_{22}=C_{33}, C_{12}=C_{23}=C_{31}$, and $c_{44}={ }_{C 55}=C_{66}$ hold, hence the elastic constant matrix $C$ can be expressed as Eq. (7).

$$
\mathrm{C}=\left(\begin{array}{cccccc}
c_{11} & c_{12} & c_{12} & 0 & 0 & 0 \\
c_{12} & c_{11} & c_{12} & 0 & 0 & 0 \\
c_{12} & c_{12} & c_{11} & 0 & 0 & 0 \\
0 & 0 & 0 & c_{44} & 0 & 0 \\
0 & 0 & 0 & 0 & c_{44} & 0 \\
0 & 0 & 0 & 0 & 0 & c_{44}
\end{array}\right)
$$

In this work, CASTEP was used to calculate the elastic constants of $\mathrm{FeAl}$ and $\mathrm{Fe}-\mathrm{Cr}-\mathrm{Al}$. Strains of ( $x, 0,0,0,0,0$ ) with $x= \pm 0.001$ and \pm 0.003 were chosen to obtain sufficient stress values and accurate elastic constants.

Table 2 lists the calculated and reference lattice parameters of FeAl and the elastic constants, bulk modulus, and shear modulus of $\mathrm{FeAl}$ and $\mathrm{Fe}-\mathrm{Cr}-\mathrm{Al}$ calculated by the GGA-PBE and LDA formulations. The bulk modulus, and shear modulus were calculated using the Hill calculation method [8], $B$ denotes the bulk moduli and $G$ denotes the shear moduli.

Table 2 Lattice parameters ( $\AA$ ), elastic constants (GPa), shear moduli (GPa) $G$ and bulk moduli (GPa) $B$ of $\mathrm{Fe}_{8} \mathrm{Al}_{8}$ and $\mathrm{Fe}_{8} \mathrm{CrAl}_{7}$.

\begin{tabular}{llllllll}
\hline & & $\begin{array}{l}\text { Lattice } \\
\text { Parameter } \\
(\AA)\end{array}$ & $\begin{array}{l}\mathrm{C}_{11} \\
(\mathrm{GPa})\end{array}$ & $\begin{array}{l}\mathrm{C}_{12} \\
(\mathrm{GPa})\end{array}$ & $\begin{array}{l}\mathrm{C}_{44} \\
(\mathrm{GPa})\end{array}$ & $\begin{array}{l}\mathrm{G} \\
(\mathrm{GPa})\end{array}$ & $B(\mathrm{GPa})$ \\
\hline & GGA-PBE & 5.71 & 274.24 & 138.5 & 151.36 & 109.7 & 183.75 \\
$\mathrm{Fe}_{8} \mathrm{Al}_{8}$ & LDA & 5.57 & 330.38 & 151.15 & 176.88 & 134.64 & 210.89 \\
& Ref. & $5.72^{10}$ & $290^{10}$ & $130^{10}$ & $166^{10}$ & & $177^{11}$ \\
$\mathrm{Fe}_{8} \mathrm{CrAl}$ & GGA-PBE & & 332.08 & 151.86 & 146.4 & 120.51 & 211.93 \\
7 & LDA & & 348.82 & 135.24 & 167.71 & 139.95 & 206.43 \\
\hline
\end{tabular}

For FeAl crystals, Table 2 shows that the LDA calculation predicts denser structures than the GGA-PBE calculations, as represented by the smaller lattice parameters. Furthermore, Table 2 also shows that the $c_{i j}$ values predicted by the LDA calculation are generally larger than those predicted by the PBE calculations. 
The elastic stabilities of the cubic crystal structures need to satisfy the mechanical equilibrium conditions under ambient conditions ${ }^{[9]}$, expressed as Eq. (8).

$$
c_{11}>0, c_{11}>\left|c_{12}\right|, c_{44}>0, c_{11}+2 c_{12}>0, c_{11}>B>c_{12}
$$

For the elastic constants listed in Table 2, the FeAl and $\mathrm{Fe}-\mathrm{Cr}-\mathrm{Al}$ structures satisfy the mechanical equilibrium standards, illustrating that these are stable structures. The good agreement between the $c_{i j}$ values of FeAl calculated by GGA-PBE and those measured experimentally ${ }^{[10]}$ shows that the GGA-PBE can be used for the Fe-Cr-Al structures. The Hill's calculation method[8] is as follows: the bulk modulus $B$ is calculated by the expression $B=\left(C_{11}+2 C_{12}\right) / 3$, the shear modulus $G$ is the average value of $G_{V}$ and $G_{R}, G_{V}$ is calculated by the expression $G_{V}=\left(C_{11}-C_{12}+3 C_{44}\right) / 5$, and $G_{R}$ is calculated by the expression $\mathrm{G}_{\mathrm{R}}=\left[5\left(\mathrm{C}_{11}-\mathrm{C}_{12}\right) \mathrm{C}_{44}\right] /\left[4 \mathrm{C}_{44}+3\left(\mathrm{C}_{11}-\mathrm{C}_{12}\right)\right]$. From Table 2 the GGA-PBE calculations predicting the bulk and shear moduli of FeAl are closer to the experimental data ${ }^{[11]}$ than the LDA calculations.

The Pugh ratio $(B / G),{ }^{[12]}$ Poisson's ratio $((3 B-2 G) / 2(3 B+G)),{ }^{[13]}$ and Cauchy pressure $\left(c_{12}-\right.$ $\left.C_{44}\right)^{[14]}$ are normally used to predict the brittleness or ductility of materials; the larger the values, the more ductile the material. In Fig. 2, the Pugh ratio, the Poisson's ratio and Cauchy pressure line charts of $\mathrm{Fe}_{8} \mathrm{Al}_{8}$ and $\mathrm{Fe}_{8} \mathrm{CrAl}_{7}$, calculated by GGA-PBE and LDA, show the same tendencies. The values calculated by LDA show that $\mathrm{Cr}$ addition lowers the ductility of FeAl, which is contradictory to the results of previous studies. ${ }^{[3]}$ In addition, large errors in the FeAl $c_{i j}$ values are obtained when the LDA method is used together with experimental and other calculated values.
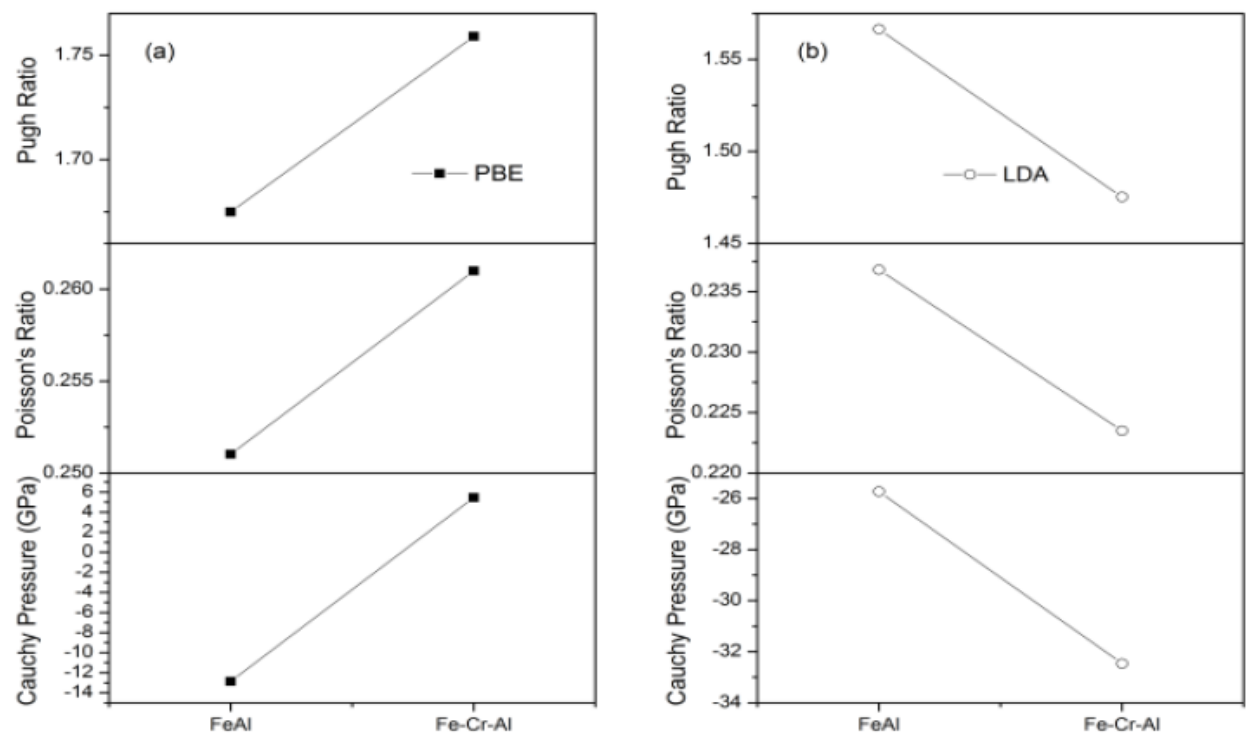

Figure 2. Pugh ratio, Poisson's ratio, and Cauchy pressure (GPa) of $\mathrm{Fe}_{8} \mathrm{Al}_{8}$ and $\mathrm{Fe}_{8} \mathrm{CrAl}_{7}$ calculated by GGA-PBE (a) and LDA (b).

The elastic properties calculated by GGA-PBE and LDA are different. $\mathrm{Cr}$ is predicted by GGA-PBE to increase the ductility of FeAl, while it is predicted by LDA to decrease the ductility of FeAl. The Fe-Cr-Al elastic constants and the elastic properties predicted by LDA deviate from the existing experimental results. ${ }^{[11]}$ In contrast, the calculations by GGA-PBE can be considered to be precise since the elastic constants and properties predicted by this method are consistent with existing research results.

\section{Electronic Properties Analysis}

In order to clarify the effects of the Cr alloying element on the electronic properties, the density of states (DOS), containing the total density of states (TDOS) and the partial density of states (PDOS), were calculated by GGA-PBE and the results are shown in Fig. 3. Fig. 2 (a) illustrates the TDOS and PDOS of Fe8Al8. In Fe8Al8, the bonding peak is mainly contributed from the valence electrons of Fe 
and $\mathrm{Al}$. The bonding originates from $\mathrm{Fe}(3 \mathrm{~d})(4 \mathrm{~s})(3 \mathrm{p})$ and $\mathrm{Al}(3 \mathrm{~s})(3 \mathrm{p})$, particularly from $\mathrm{Fe}(3 \mathrm{~d})$. From the DOS of FeAl alloys, the bonding property can be considered mainly a valence property, illustrating that this is the reason for the brittleness of FeAl. Fig. 2 (b) displays the DOS of Fe8CrAl7, in which three the bonding peaks occur, two of which originate from $\mathrm{Cr}(4 \mathrm{~s})$ and $\mathrm{Cr}(3 \mathrm{p})$ under the fermi energy level. The bonding peak is mainly contributed from the valence electrons of Fe, $\mathrm{Al}$, and $\mathrm{Cr}$. The hybrid bonding mainly originates from $\mathrm{Fe}$ (3d ) and $\mathrm{Cr}$ (3d). The number of bonding peaks of Fe8CrAl7 is greater than that of Fe8Al8, which can be considered one result of the improved cohesive capacity of FeAl caused by Cr.
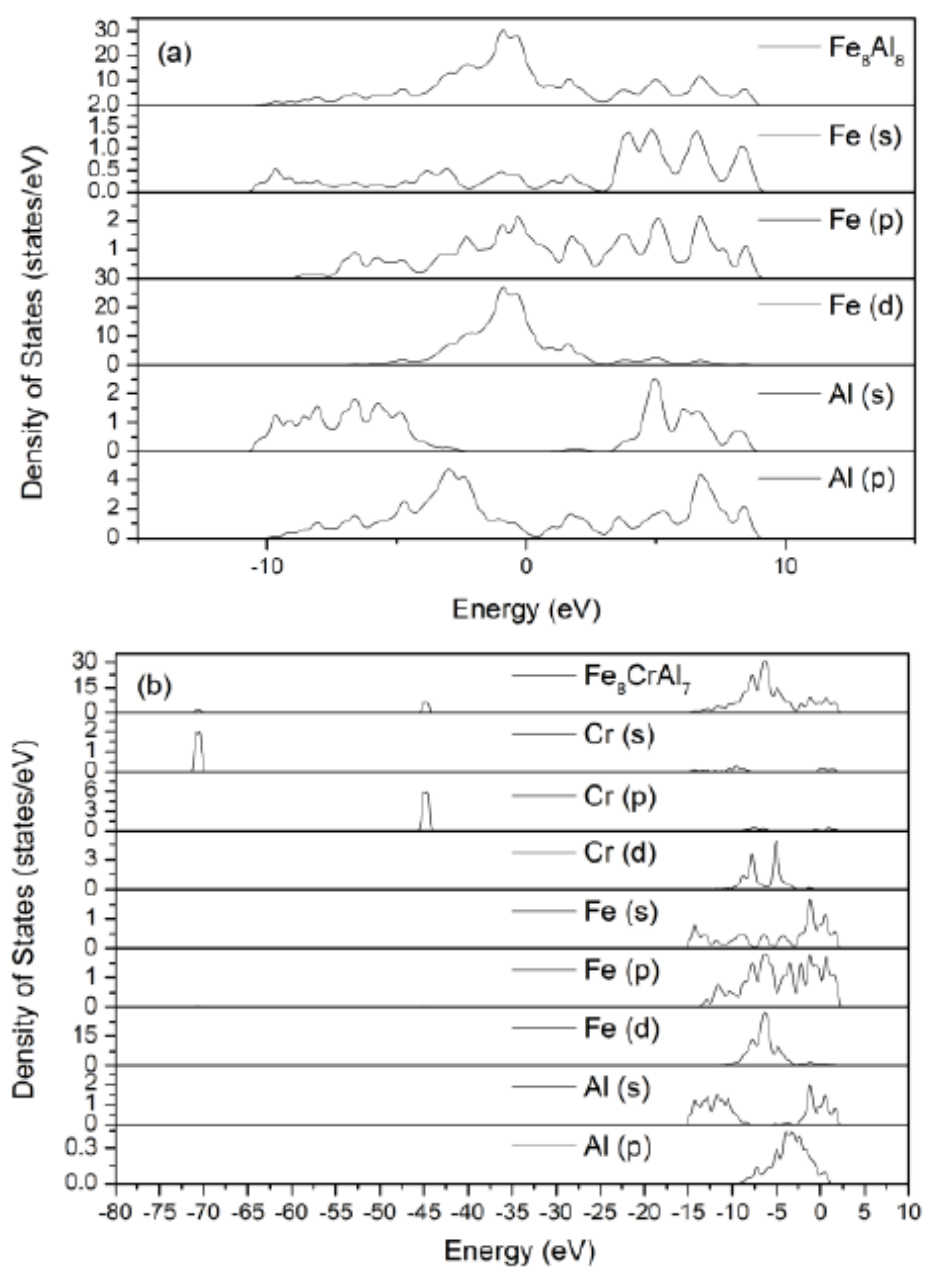

Figure3. Density of states of $\mathrm{Fe}_{8} \mathrm{Al}_{8}$ (a) and $\mathrm{Fe}_{8} \mathrm{CrAl}_{7}$ (b)

\section{Electron Densities Analysis}

Difference electron density can be performed to visually illustrate the electron configuration. Fig. 4 (a) and (b) display the difference electron densities in the (110) plane for Fe8Al8 and Fe8CrAl7, calculated by the GGA-PBE method. In Fe8CrAl7, Cr rearranges the electron distribution near the Fe and $\mathrm{Al}$ atoms, and the overlapped electrons between $\mathrm{Cr}$ and $\mathrm{Fe}$ are heavier than those between $\mathrm{Al}$ and Fe in Fe8Al8. The results are compatible with the above analysis of elastic properties and DOS. 


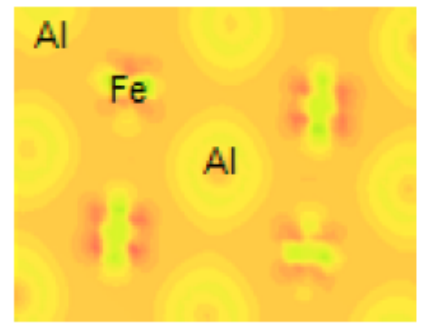

(a)

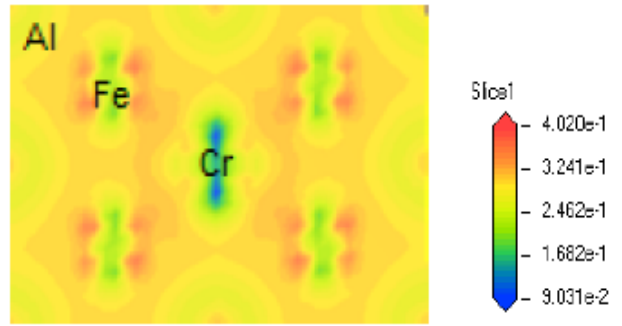

(b)

Figure 4. Difference electron densities of (110) plane in $\mathrm{Fe}_{8} \mathrm{Al}_{8}(\mathrm{a})$ and $\mathrm{Fe}_{8} \mathrm{CrAl}_{7}(\mathrm{~b})$.

\section{Conclusions}

(1) The elastic constants cij of B2-phase FeAl intermetallic compounds and Fe-Cr-Al computed using the strain-stress method with GGA-PBE is more accurate than those computed using LDA.

(2) Alloy elements such as Cr increase the ductility of FeAl.

(3) The alloying elements affect the $\boldsymbol{c}_{\boldsymbol{i j}}$ values of FeAl as they affect the density of states and electron density.

\section{Acknowledgements}

This work was financially supported by the National Natural Science Foundation of China (Grant No.51371097), by A Project Funded by the Priority Academic Program Development of Jiangsu Higher Education Institutions, by Qing Lan Project, by Jiangsu Precision Manufacturing Engineering Technology Research and Development Center Foundation of China (Grant No.ZK13-02-02).

\section{References}

[1] N.S. Stoloff: 'Iron aluminides: present status and future propects', Materials Science and Engineering A, 1998, 258, 1-14.

[2] M. Palm: 'Concepts derived from phase diagram studies for the strengthening of Fe-Al-based alloys’, Inermetallics, 2005, 13, 1286-1295.

[3] J. H. Schneibel, E. D. Specht and W.A. Simpson: 'Solid solution strengthening in ternary B2 iron aluminides containing 3d transition elements’, Intermetallics, 1996, 4, 581-583.

[4] M. D. Segall, P. L. Lindan, M. J. Probert, CJ Pickard, P. J. Hasnip, S.J. Clark and M.C. Payne: 'First-principles simulation:Ideas,illustrations and the CASTEP code', J. Phys. Condens. Matter., 2002, 14, 2717-2743.

[5] J. P. Perdew, K. Burke and M. Ernzerhof: 'Generalized Gradient Approximation Made Simple' Phys. Rev. Lett., 1996, 77, 3865-3868.

[6] D. M. Ceperley and B. J. Alder: 'Ground State of the Electron Gas by a Stochastic Method', Phys. Rev. Lett. 1980, 45 566-569.

[7] Shunli Shang, Yi Wang, and Zi-Kui Liu: 'First-principles elastic constants of $\alpha$ - and $\theta-\mathrm{Al} 2 \mathrm{O3}$ ' Appl. Phys. Lett., 2007, 90, 101909-101911.

[8] Hill R.: 'The elastic behaviour of a crystalline aggregate’, Proc. Phys. Soc. A, 1952, 65, 349.

[9] O. Beckstein, J. Klepeis, G. Hart and O. Pankratov: 'First-principles elastic constants and electronic structure of $\alpha-\mathrm{Pt} 2 \mathrm{Si}$ and PtSi', Phys. Rev. B, 2001, 63,134112.

[10] B. Zhang and WA. Soffa: 'The structure and properties of L10 ordered ferromagnets:Co-Pt, FePt, FePd and MnAl', Scr. Metall. Mater., 1994, 30, 683-688. 
[11] M. MJ and K. BM: 'Intermetallic compounds, principles \& practices', John Wiley \& Sons Publisher: London. pp. 195, 1994.

[12] S.F. Pugh: 'Relations between the elastic moduli and the plastic properties of polycrystalline pure metials’, Philos Mag., 1954, 45, 823-843.

[13] R. H. Baughman, J. M. Shacklette, A. A. Zakhidov, et al.: 'Negative Poisson's ratios as a common feature of cubic metals', Nature, 1998, 392, 362-365.

[14] D. G. Pettifor: 'Theoretical predictions of struture and related properties of intermetallics', Mater. Sci. Technol., 1992, 8, 345-349. 